\title{
Patients' Perception and Residents' Self-evaluation of the Communication Skills of Obstetrics and Gynecology Residents
}

\author{
Abrar A. Qari ${ }^{1,2,3}$, Muath M. Alswat ${ }^{2,4, *}$ (D), Bashayer S. Alharbi ${ }^{2,4}$, Asma Khalil ${ }^{1,2,3}$ \\ ${ }^{1}$ Department of Obstetrics and Gynecology, Ministry of the National Guard-Health Affairs, Jeddah, Saudi Arabia \\ ${ }^{2}$ King Abdullah International Medical Research Center, Jeddah, Saudi Arabia \\ ${ }^{3}$ Deanship of Postgrad Education, King Saud bin Abdulaziz University for Health Sciences, Jeddah, Saudi Arabia \\ ${ }^{4}$ College of Medicine, King Saud bin Abdulaziz University for Health Sciences, Jeddah, Saudi Arabia
}

\section{ARTICLE INFO \\ Article History \\ Received 02 May 2020 \\ Accepted 22 August 2020 \\ Keywords \\ Communication \\ residency \\ obstetrics and gynecology \\ physician-patient relations \\ Saudi Arabia}

\begin{abstract}
The objective of this study was to assess the patients' perceptions of the communication skills of obstetrics and gynecology residents, highlighting areas where these skills could be improved, and comparing the patient perceptions with the residents' self-evaluation. This was a cross-sectional study, conducted in King Abdulaziz Medical City in Jeddah, Saudi Arabia. Data were collected from patients attending the obstetrics and gynecology clinics and consulted with a resident. The required study sample was collected within a 3-month period. The Arabic translated and validated version of the Communication Assessment Tool (CAT) was used. A total of 179 patients completed the CAT. The mean age of the participants was 32 years. The mean time spent with a resident in the consultation was $22.1 \pm 11.8 \mathrm{~min}$, and the mean percentage of an excellent rating was $79.67 \%$. The item most frequently rated as excellent was "Treated me with respect" (89.5\%). The item rated least frequently as excellent was "Involved me in decisions as much as I wanted" (72.1\%). Comparing training levels, there were no significant difference found in the overall percentage of items rated as excellent or for the items of the CAT $(p=0.406)$. The time spent with patients was also not significant $(p=0.069)$. The overall mean percentage of items rated by the residents as "excellent" was $69.91 \%$, lower than the patients' perception. The finding of the study is considered a benchmark for obstetrics and gynecology residency programs to compare their performance with other centers.
\end{abstract}

(C) 2020 Dr. Sulaiman Al Habib Medical Group. Publishing services by Atlantis Press International B.V. This is an open access article distributed under the CC BY-NC 4.0 license (http://creativecommons.org/licenses/by-nc/4.0/).

\section{INTRODUCTION}

Effective communication has a significant impact on patient related health promotion and health education, through improving patient compliance, satisfaction, and psychological well-being, that is, less fear or anxiety. The main goals of physician-patient interaction are sharing information to aid in the diagnosis and treatment, building a healthy relationship, and education [1-6]. The communication skills of physicians can also influence the active involvement of patients in their care [1]. In a study conducted in Tabuk, patient compliance with medication was affected by the communication with their physicians, and about $20 \%$ of those who were noncompliant attributed that to miscommunication [7]. Another aspect that is highly affected by patient-physician communication is patient satisfaction. It has been shown that good patient-physician communication is related with patient satisfaction [8]. Moreover, interpersonal and communication skills have been identified as one of the six general competencies for physicians by the Accreditation Council for Graduate Medical Education and the American Board of Medical Specialties [9]. However, in Saudi Arabia communication

"Corresponding author. Email: alswat016@ksau-hs.edu.sa

Peer review under responsibility of the Dr. Sulaiman Al Habib Medical Group

Data availability statement: The data that support the findings of this study are available

from the corresponding author, [Alswat, $\mathrm{M}]$, upon reasonable request. skills are hardly included in any undergraduate or postgraduate medical curriculum. There is also scarcity of research in this area.

Assessing the level of communication skills from the perspective of patients and physicians facilitates the identification of the gap between patient needs and the perceptions of physicians and in this way, helps improve the healthcare services provided $[10,11]$. Eliciting the agenda of the patient with open-ended questions, understanding the patient's perspective of their illness, expressing empathy, and shared decision-making are key features of patient-centered communication skills essential for physicians to deliver high-quality healthcare services [12]. However, measuring the levels of communication skills is challenging because the patients' needs should be clearly defined $[11,13,14]$.

The Communication Assessment Tool (CAT), developed by Makoul et al. [9], is a valid and reliable method to assess the patients' perceptions of the interpersonal and communication skills of physicians. Specifically, the tool developers reviewed prominent models to generate a list of communication tasks and conducted focus groups to gather patient perspectives on items and response scales; in addition, a national survey was conducted to determine the importance of each item and an expert review was performed to ensure a comprehensive list of items. A Lexile analysis was done to assess the readability, which corresponds to a fourth grade reading level, and a psychometric analysis was performed to determine 
the most viable response scale [9]. In comparison with other assessment tools that depend on recalling events over a period or combining communications skills and treatment satisfaction, the advantage of the CAT is that the patients are asked to concentrate on an encounter they just had with the physician, and this direct capture provides focused feedback to training physicians.

Many studies have been done related to physician-patient communication [15]. In a study investigating the communication skills of family medicine residents, the authors reported that residents with a higher level of training had lower scores than junior residents. They attributed the finding to the longer time junior residents spent with each patient [16]. Although communication is an important aspect of patient care and an essential part of the residency training program, few studies explored the communication skills of residents and physicians while in training. To the best of our knowledge, this is the first study to explore obstetrics and gynecology practice. The aim of this study was to assess the communication skills of obstetrics and gynecology residents to provide a framework for feedback to the residents and enhance their communication based on the aspects highlighted in the results. The findings will be disseminated to benefit the communication skills of obstetrics and gynecology residents regionally and nationally.

\section{METHODOLOGY}

\subsection{Study Design and Setting}

This cross-sectional study was conducted at the obstetrics and gynecology outpatient clinics in King Abdulaziz Medical City in Jeddah, Saudi Arabia, from March to June 2019.

\subsection{Study Population}

Patients attended by a resident at any level in the obstetrics and gynecology outpatient clinics were included. Volunteer medical students invited the patient to complete the questionnaires, and calculated the consultation time. Patients were informed that their participation is voluntary, confidential, and anonymous, and that it will not affect their treatment plan in any way. The completed questionnaires were submitted to the registry office without any resident access. The exclusion criteria were as follows: disability such as deafness, psychiatric and mentally ill patients, emergency cases (e.g., in labor or severe or active vaginal bleeding); patients seen by non-obstetrics and gynecology rotating residents; illiterate patients. Sample size was calculated considering the following: confidence level 95\%, population size (obstetrics and gynecology residents in the Western Region): 312, margin of error $5 \%$, and the calculated sample size was 173 patients. The CAT questionnaire was conveniently distributed to patients attending the morning and afternoon clinics managed by obstetrics and gynecology residents from Sunday to Thursday. The same questionnaire was distributed to the residents after the patient sample was completed to compare their self-evaluation to the patient responses.

\subsection{The CAT}

The CAT assesses 14 items using a 5-point Likert rating scale (where 1 = poor, 2 = fair, 3 = good, 4 = very good, $5=$ excellent).
When testing the validity and reliability, the 15-item CAT was internally consistent, with a high scale reliability (Cronbach's coefficient alpha $=0.98$ ) [9]. The CAT Arabic version from the Alsaad et al. [6] study was used, which was based on the World Health Organization criteria and translated. The translated tool was piloted and found suitable for patients in Saudi Arabia. The demographic data of the patient including age and level of education was collected as well as the resident's level of education.

\subsection{Statistical Analyses}

Data entry and analysis was performed using SPSS software (Statistic Package for Social Sciences, Version 22; SPSS Inc., Chicago, IL, USA). Categorical data are presented as frequency and percentage. The proportion of items rated as excellent was calculated based on the total number of items of the CAT questionnaire. The overall mean score out of 5 was also calculated. As the data were not normally distributed, a nonparametric analysis was performed. The Kruskal-Wallis one-way analysis of variance test was used for the comparison of the overall score and items rated as excellent by year of training. For comparing the items rated as excellent after the first visit to the resident or not, a Mann-Whitney $U$-test was used. The correlation between the time spent with the patient and the percentage of items rated as excellent was tested using the Pearson $r$-test. A chi-square test was used to compare the groups of patients and residents. A $p$-value $<0.05$ was considered statistically significant.

\subsection{Ethical Considerations}

The study was approved by the Institutional Ethics Review Board of King Abdullah International Medical Research Center. Informed consent was obtained from patients and residents. No data that could identify the residents and patients such as a name or medical record number were collected.

\section{RESULTS}

Data were collected from outpatients managed by obstetrics and gynecology residents at King Abdulaziz Medical City in Jeddah. A total of 179 participants were included within 12 weeks from February 2019. No missing data were reported in the completed questionnaires. The mean age of the participants was 32 years, and more than half $(54.7 \%)$ achieved a bachelor's degree. The mean time spent with a resident in the consultation was $22.1 \pm 11.8 \mathrm{~min}$. More information is available in Table 1.

The overall mean of the rating score was $4.708 \pm 0.076$, and the overall mean percentage of excellent scores was $79.67 \%$. The items frequently rated as excellent were "Treated me with respect" (89.5\%), "Showed care and concern" (86\%), and "Let me talk without interruptions" (85.5\%). In contrast, the items least frequently rated as excellent were "Involved me in decisions as much as I wanted" (72.1\%), "Encouraged me to ask questions" (76.2\%), and "Checked to be sure I understood everything" (79.7) (Table 2). The overall percentage of items scored as excellent were not statistically significant in terms of the year of training $\left(\chi^{2}=4,4 d f\right.$, $p=0.406)$, and the time spent with patients was also not significant $(p=0.069)$; details are shown in Table 3 . 
There was no significant difference found in the overall percentage of items rated as excellent when comparing patients who were seen by the resident previously $(81.58 \%, n=124)$ and patients not seen by the resident before $(71.93 \%, n=34)(U=1850.5, z=-1.149, p=0.251)$.

All obstetrics and gynecology residents completed the CAT questionnaire to self-evaluate their communication skills. There were

Table $1 \mid$ Demographic characteristics of patients $(n=179)$

\begin{tabular}{lccc}
\hline & Mean (SD) & Minimum & Maximum \\
\cline { 2 - 4 } Age & $\mathbf{3 2 . 2 9 ( 7 . 1 )}$ & $\mathbf{2 0}$ & $\mathbf{6 3}$ \\
\cline { 2 - 4 } & & $\mathbf{\%}$ & $\boldsymbol{n}$ \\
\hline Education level & & \\
High & 2.5 & 4 \\
Bachelor & 56.7 & 89 \\
Secondary school & 31.8 & 50 \\
Less & 8.9 & 14 \\
Seen by the same resident before & & \\
$\quad$ Yes & 20.9 & 33 \\
$\quad$ No & 77.8 & 123 \\
\hline
\end{tabular}

SD, standard deviation.

Table 2 Percentage of excellent ratings for individual CAT items overall (patients' answers)

\begin{tabular}{llc}
\hline CAT question & $\begin{array}{c}\text { \% Items } \\
\text { scored as } \\
\text { excellent }\end{array}$ \\
\hline 1 Greeted me in a way that made me feel comfortable & 77.9 \\
2 & Treated me with respect & 88.9 \\
3 & Showed interest in my ideas about my health & 80.8 \\
4 & Understood my main health concerns & 79.1 \\
5 & Paid attention to me (looked at me, listened carefully) & 83.1 \\
6 & Let me talk without interruptions & 85.5 \\
7 & Gave me as much information as I wanted & 77.9 \\
8 & Talked in terms I could understand & 83.7 \\
9 & Checked to be sure I understood everything & 76.7 \\
10 & Encouraged me to ask questions & 76.2 \\
11 & Involved me in decisions as much as I wanted & 72.1 \\
12 & Discussed next steps, including any follow-up plans & 81.4 \\
13 & Showed care and concern & 86.0 \\
14 & Spent the right amount of time with me & 84.3 \\
\hline
\end{tabular}

25 residents. Regarding their residency levels, $20 \%$ were R1, 20\% were R2, 36\% were R3, $12 \%$ were R4, and $12 \%$ were $\mathrm{R} 5$. The mean rating score was $4.737 \pm 0.592$. The overall mean percentage of the items the residents rated as "excellent" was $69.91 \%( \pm 16.85)$, which was lower than the patients' perception. The three items rated most frequently as excellent by the residents were "Greeted patient in a way that made her feel comfortable" (100\%), "Treated patient with respect" (89.94\%), and "Showed care and concern" (86.03\%). The items rated least frequently as excellent were "Encouraged patient to ask questions" (33.3\%), "Let patient talk without interruptions" (48\%), and "Understood the patient's main health concerns" (58.3\%) (Figure 1).

\section{DISCUSSION}

This study assessed patients' perceptions of the communication skills of obstetrics and gynecology residents in King Abdulaziz Medical City in Jeddah. The CAT questionnaire was used to measure the patients' perceptions, and the instrument was deemed suitable to assess the patients' perception of the residents [12]. No current literature is available with the same specialty (obstetrics and gynecology) for comparison. The current study is considered a benchmark for future studies in the same specialty.

The overall mean percentage of the CAT items rated by the patients as "excellent" was $79.67 \%$, similar to that reported by

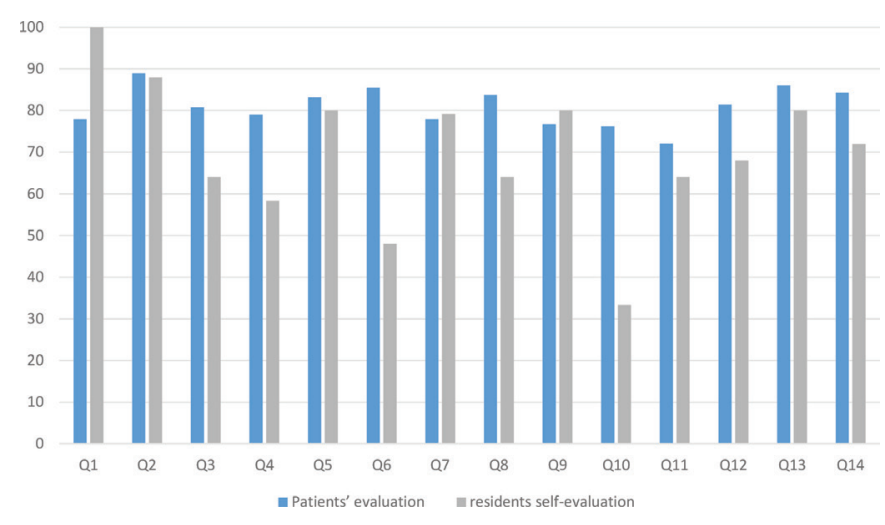

Figure 1 Comparison of percentage of excellent ratings between patients' evaluation and residents' self-evaluation.

Table 3 Percentage of excellent ratings for each residency level overall (patients' answers)

\begin{tabular}{|c|c|c|c|c|c|}
\hline CAT item & R1 & R2 & R3 & R4 & R5 \\
\hline 1. Greeted me in a way that made me feel comfortable & 93.8 & 82.4 & 73.3 & 93.3 & 69.7 \\
\hline 2. Treated me with respect & 100 & 88.2 & 86.7 & 100 & 84.8 \\
\hline 3. Showed interest in my ideas about my health & 81.2 & 76.5 & 77.3 & 93.3 & 78.8 \\
\hline 4. Understood my main health concerns & 75 & 76.5 & 77.3 & 86.7 & 72.7 \\
\hline 5. Paid attention to me (looked at me, listened carefully) & 80 & 93.8 & 81.3 & 91.7 & 75 \\
\hline 6. Let me talk without interruptions & 93.3 & 87.5 & 84 & 91.7 & 81.2 \\
\hline 7. Gave me as much information as I wanted & 86.7 & 75 & 77.3 & 66.7 & 71.9 \\
\hline 8. Talked in terms I could understand & 93.3 & 81.2 & 82.7 & 75 & 81.2 \\
\hline 9. Checked to be sure I understood everything & 93.3 & 81.2 & 70.7 & 75 & 71.9 \\
\hline 10. Encouraged me to ask questions & 86.7 & 87.5 & 69.3 & 75 & 78.1 \\
\hline 11. Involved me in decisions as much as I wanted & 73.3 & 75 & 65.3 & 75 & 71.9 \\
\hline 12. Discussed next steps, including any follow-up plans & 86.7 & 87.5 & 76 & 83.3 & 81.2 \\
\hline 13. Showed care and concern & 100 & 94.1 & 82.7 & 93.3 & 81.8 \\
\hline 14. Spent the right amount of time with me & 100 & 87.5 & 80 & 91.7 & 78.1 \\
\hline
\end{tabular}

$\mathrm{R}$, residency level. 
Alsaad et al. [6] and Myerholtz et al. [16], both at 71\%. This finding can possibly be attributed to a higher level of training of the residency as most of the patients were seen by senior residents. However, the residency level was not a statistically significant factor. Myerholtz et al. [16] reported that the 1st-year training residents scored higher, which correlated with the time spent with patients. These findings were in contrast to the expectation that residents with more training and experience would receive higher patient ratings. We found no significant difference in the time spent with patients and an excellent score.

The "Treated me with respect" item, rated most frequently as excellent in our study (89.5\%), also scored the highest in the study of Alsaad et al. [6] and Myerholtz et al. [16] (82.3\% and 72.8\%, respectively). This was also the case for the items rated least frequently as excellent. The items "Involved me in decisions as much as I wanted" (72.1\%) and "Encouraged me to ask questions" (76.2\%) scored similarly in Makoul et al.s [9] report and were the lowest-rated items for family medicine residents in the study of Alsaad et al. [6] and Myerholtz et al. [16].

The first few minutes in the beginning of the interview are crucial because the foundation of the interview is created within them [17]. It has been noticed in this study that residents were rated higher in the items that occur in the beginning of the interview such as greeting the patients, treating them with respect, and not interrupting them. By contrast, items occurring at the end of the interview-such as making sure the patients understood everything, encouraging them to ask questions, and involving them in decisions-were rated lower. This may happen because the residents are getting ready for the next patient or activity, which was also observed in another study [18].

The overall mean percentage of the CAT items rated by the residents as "excellent" was $69.91 \%$, which was lower than the patients' perception (81\%), not supporting the perception that physicians tend to score themselves higher compared with patients [11]. In addition, the items most and least frequently rated as "excellent" by the residents were similar to the patients' perception about the residents. It appears that the patients perceived the residents as caring and respectful and allowed them to express themselves without interruptions. However, the patients appeared to be less satisfied with their active involvement in the decision-making process.

The results of the study are limited by the use of a single-center approach and a limited sample size. There was also a varying amount of data collected for each resident owing to differences in the number of patients seen per resident, which may limit the stability and generalizability of the data. However, we maintained a minimum of 15 questionnaires per resident, as the Rasch generalizability theory "estimates that 12-30 ratings per examinee are required when seeking a reliability of 0.96 for data collected on a 5 -point scale" to be confident that the results will give a true picture of patient perceptions of the resident's communication skills [11]. Future studies may want to ensure a minimum of 20 questionnaires per resident to increase the generalizability of data.

Additional research is required to increase the current understanding of patient-doctor communication and its impact on patient outcomes. Future studies could target the key elements described in this paper and explore their influence, to facilitate the design and implementation of appropriate educational programs for training physicians. Few studies explored the relationship between gender and the perceptions of residents' communication skills, and gender could be explored in future studies.

To the best of the authors' knowledge, this is the first paper to evaluate the communication skills of residents from the perspective of both patients and training residents in the obstetrics and gynecology specialty in Saudi Arabia. To develop normative data for the CAT in the obstetrics and gynecology residency program, future studies should increase the number of participating programs.

\section{CONCLUSION}

Using the CAT to assess the communication skills of obstetrics and gynecology residents highlighted a high excellent rating score from the patients' perspective. This tool can be used to provide direct structured feedback to the residents and continuity of selfevaluation to improve areas where improvement is required. In addition, group feedback and workshops can be conducted to highlight the least scoring items, which will facilitate learning processes during the residency training period. The study results can be considered a benchmark to compare with other obstetrics and gynecology residency program centers in Saudi Arabia.

\section{CONFLICTS OF INTEREST}

The authors declare they have no conflicts of interest.

\section{AUTHORS' CONTRIBUTION}

All authors have contributed to the manuscript and fulfilled the authorship criteria. AAQ and MMA were responsible for conception and design of the study. MMA and BSA provided statistical expertise and managed the analysis and interpretation of the data. AAQ, MMA, BSA and AK participated in drafting the article AAQ, MMA and BSA provided critical revision of the article for important intellectual content. MMA and BSA participated in collection and assembly of the data. All authors approved the final version to be submitted.

\section{ACKNOWLEDGMENTS}

We acknowledge Dr Sulaiman A. Alshammari and his team for allowing us to use the Arabic translated CAT from their study. We also thank the data collectors, residents, and patients, whose participation enabled us to complete this study.

\section{REFERENCES}

[1] Boerebach BCM, Scheepers RA, van der Leeuw RM, Heineman MJ, Arah OA, Lombarts KMJMH. The impact of clinicians' personality and their interpersonal behaviors on the quality of patient care: a systematic review. Int J Qual Health Care 2014;26;426-81.

[2] Elzubier AG. Doctor-patient communication: a skill needed in Saudi Arabia. J Family Community Med 2002;9;51-6. 
[3] Simpson M, Buckman R, Stewart M, Maguire P, Lipkin M, Novack D, et al. Doctor-patient communication: the Toronto consensus statement. BMJ 1991;303;1385-7.

[4] Arora NK. Interacting with cancer patients: the significance of physicians' communication behavior. Soc Sci Med 2003;57; 791-806.

[5] Fawole OA, Dy SM, Wilson RF, Lau BD, Martinez KA, Apostol CC, et al. A systematic review of communication quality improvement interventions for patients with advanced and serious illness. J Gen Intern Med 2013;28;570-7.

[6] Alsaad SM, Alshammari SA, Almogbel TA. Appraisal of the communication skills of residents in the Family Medicine Program in Central Saudi Arabia. Saudi Med J 2016;37;804-8.

[7] Khalil SA, Elzubier AG. Drug compliance among hypertensive patients in Tabuk, Saudi Arabia. J Hypertens 1997;15;561-5.

[8] Burgener AM. Enhancing communication to improve patient safety and to increase patient satisfaction. Health Care Manag (Frederick) 2017;36;238-43.

[9] Makoul G, Krupat E, Chang CH. Measuring patient views of physician communication skills: development and testing of the Communication Assessment Tool. Patient Educ Couns 2007;67;333-42.

[10] Cegala DJ, Gade C, Broz SL, McClure L. Physicians' and patients' perceptions of patients' communication competence in a primary care medical interview. Health Commun 2004;16;289-304.
[11] Yasein NA, Shakhatreh FM, Shroukh WA, Farah MS, Jaber RM. A comparison between patients' and residents' perceptions of patient centeredness and communication skills among physicians working at Jordan University Hospital. Open J Nurs 2017;7; 698-706.

[12] Hashim MJ. Patient-centered communication: basic skills. Am Fam Physician 2017;95;29-34.

[13] Clayton MF, Latimer S, Dunn TW, Haas L. Assessing patientcentered communication in a family practice setting: how do we measure it, and whose opinion matters? Patient Educ Couns 2011;84;294-302.

[14] Schattner A, Rudin D, Jellin N. Good physicians from the perspective of their patients. BMC Health Serv Res 2004;4;26.

[15] Skelton JR, Murray J, Hobbs FD. Imprecision in medical communication: study of a doctor talking to patients with serious illness. J R Soc Med 1999;92;620-5.

[16] Myerholtz L, Simons L, Felix S, Nguyen T, Brennan J, RiveraTovar A, et al. Using the communication assessment tool in family medicine residency programs. Fam Med 2010;42;567-73.

[17] Berman AC, Chutka DS. Assessing effective physician-patient communication skills: "are you listening to me, doc?". Korean J Med Educ 2016;28;243-9.

[18] Rahman A, Tasnim S. Twelve tips for better communication with patients during history-taking. Scientific World Journal 2007;7;519-24. 